\title{
Coronary artery calcium or epicardial fat: Different markers for different people
}

\author{
Nataliya Pyslar, MD, a and Rami Doukky, MD, MSc, MBA, FASNC ${ }^{\mathrm{a}, \mathrm{b}}$ \\ a Division of Cardiology, Cook County Health, Chicago, IL \\ b Division of Cardiology, Rush University Medical Center, Chicago, IL
}

Received Mar 16, 2021; accepted Mar 16, 2021

doi: $10.1007 / \mathrm{s} 12350-021-02609-7$

\section{See related article, pp. 1583-1592}

Body fat is a metabolically active endocrine organ. Functional abnormalities of adipose tissue, known as adiposopathy, may result in systemic and local endocrine and immune derangements. ${ }^{1}$ Abdominal visceral adiposopathy is notorious for the development of insulin resistance, diabetes mellitus, hepatic steatosis, metabolic syndrome and dyslipidemia, indirectly leading to coronary artery disease (CAD). ${ }^{2}$ The epicardial adipose tissue (EAT) is a unique fat compartment between the myocardium and the visceral pericardium, sharing a common embryologic origin with the visceral fat depot. ${ }^{3}$ Metabolic imbalances between adiponectin/leptin secretion by EAT may lead to coronary endothelial dysfunction and increased TNF- $\alpha$ production, triggering systemic inflammation and oxidative stress. Both abdominal visceral fat and EAT have a higher release of inflammatory cytokines than subcutaneous fat. In patients undergoing coronary artery bypass grafting (CABG), Mazurek et al demonstrated increased levels of inflammatory mediators in surgically excised EAT compared to subcutaneous fat from the lower extremities. ${ }^{4}$ Others analyzed the expression of inflammatory markers in EAT biopsies from CABG patients and concluded that epicardial and omental fat exhibit a broadly comparable pathogenic messenger ribonucleotide acid (mRNA) profile, suggesting macrophage infiltration into the EAT. These studies indicate that local chronic inflammation in EAT may contribute to the

Reprint requests: Rami Doukky, MD, MSc, MBA, FASNC, Division of Cardiology, Cook County Health, Chicago, IL; rami_doukky@ rush.edu

J Nucl Cardiol 2022;29:1593-5.

$1071-3581 / \$ 34.00$

Copyright (C) 2021 American Society of Nuclear Cardiology. pathogenesis of CAD through inflammatory mediators acting via paracrine or vasocrine pathways. ${ }^{5,6}$

Conveniently, the epicardial fat volume (EFV) can be estimated from non-contrast $\mathrm{CT}$ in patients undergoing myocardial perfusion imaging (MPI) using SPECT/CT or PET/CT. In addition, these hybrid systems yield valuable quantification of coronary artery calcium (CAC). Although the complementary diagnostic, prognostic, and decision-guiding value of CAC in patients undergoing MPI is well established, ${ }^{7}$ the clinical utility of EAT is much less studied and understood.

There are significant differences in EFV based on patient demographics. In fact, age, gender, body weight, and ethnicity seem to be major determinants of EFV. It has been shown that EFV increases with age and body mass index. Furthermore, there are some ethnic differences in epicardial and pericardial fat thickness, as nonHispanic White men have more epicardial and pericardial fat than African Americans. ${ }^{3}$ Similarly, there are significant differences in EFV between South Asian, East Asian, and Caucasian populations residing in Australia, with the highest EFV observed in South Asians and the lowest in Caucasians. ${ }^{8}$ In the multi-ethnic study of atherosclerosis (MESA), EFV was substantially lower in women than men after adjusting for age, height, weight, and study site. Among women, Chinese had higher EFV than Hispanics and Whites, while there were no significant differences among men from these ethnic groups. ${ }^{9}$

As is the case with EAT, the expression of CAC can vary among ethnic groups. In western populations, CAC score (CACS) of zero is a powerful negative risk marker in asymptomatic patients. In symptomatic patients with CACS of zero, non-calcified plaques are observed in only $1 \%-2 \%$ of patients who maintain excellent prognosis. Notably, MESA reported the lowest CACS in Chinese compared to other ethnic groups. ${ }^{10}$ In a Chinese cohort, Zhou et al demonstrated poor incremental discriminatory value for CACS when added to traditional 
risk factors, especially in patients with intermediate to high pretest probability. ${ }^{11}$ In another cohort of 5743 Chinese patients, the same group reported suboptimal performance of CACS, as $12 \%$ of patients with obstructive CAD had CACS of zero, a far greater proportion than western populations. ${ }^{12}$

In this issue of the journal, $\mathrm{Yu}$ et al reported on a retrospective cohort of 204 Chinese patients with suspected CAD who had undergone CACS and gated rest/ stress SPECT MPI. ${ }^{13}$ Following the stress MPI acquisition, a low-dose cardiac CT was performed for the quantification of CACS and EFV. Myocardial ischemia was defined as summed difference score (SDS) of $\geq 2$. CAC was quantified according to the Agatston method. Software-assisted EFV measurement was derived from the sum of cross-sectional areas of epicardial fat (intensity range of -190 to $-30 \mathrm{HU}$ ) multiplied by slice thickness. The Youden index was used to define optimal CACS (Agatston score of $>14$ ) and EFV $\left(>119.1 \mathrm{~cm}^{3}\right)$ cutoffs. Univariate and multivariable logistic regression analyses identified male gender, hypertension, CACS and EFV as significant predictors of myocardial ischemia. Receiver-operator characteristic (ROC) analysis demonstrated that the prediction of myocardial ischemia improved when high CACS was added to a model of traditional risk factors (AUC 0.74 vs $0.69, P=0.05$ ). The addition of high EFV to the model further improved the prediction of myocardial ischemia beyond traditional risk factors and CACS (AUC 0.82 vs $0.74, P=0.002$ ), with a net reclassification improvement (NRI) index of $0.14(P=0.04)$. The authors concluded that in Chinese patients with suspected CAD, EFV provides improved prediction of myocardial ischemia above and beyond traditional risk factors and CACS.

There are a few shortcomings of the current study. The small sample size, single-center design, and lack of external validation are obvious limitations. Furthermore, the study findings may only apply to Chinese population residing in China. The authors chose to explore the correlation of EFV with myocardial ischemia, but the association with other myocardial perfusion abnormalities, such as ischemia plus infarction (summed stress score), would be of interest. Importantly, the study did not report on the association between EFV and cardiovascular outcomes. Additionally, the present method of EFV quantification is time-consuming and the separation between EAT and other precordial adipose tissue is not simple. A wide clinical use of EFV would require the development of automatic quantification tool, akin of CACS measurement software.

Despite EFV being the key point of the study, CACS did improve the prediction of myocardial ischemia, albeit to a lesser degree than EFV. A potential explanation for the marginal discriminatory capacity of
CACS in this study could be the small sample size or the low threshold used to define high CACS $(>14)$. However, one should consider the possibility of low performance of CACS in Chinese subjects. Another hypothesis is that the observed ischemia could be caused by coronary microvascular and epicardial vascular dysfunction in subjects without obstructive CAD, which would explain ischemia in the face of low CACS. Finally, CACS cannot reliably serve as a diagnostic tool for obstructive CAD in symptomatic subjects, as the correlation between CACS and luminal stenosis is poor. ${ }^{14}$

The present report illustrates the adverse impact of EAT, manifesting as myocardial ischemia. Moreover, the current paper draws attention to potential differences in the burden and clinical utility of CACS and EAT among various ethnic groups. The study poses the question as to whether EAT is a better risk predictor in Chinese, at least for those residing in China, while CACS continues to be a strong indicator of risk in western populations. With the development of standardized software, EFV quantification can provide valuable adjunct data that is easily attainable in patients undergoing MPI using SPECT/CT and PET/CT systems. Future research may reveal the potential value of EAT not only in the prediction of myocardial ischemia, but also in identifying patients at risk for adverse cardiovascular events. Lastly, additional studies are needed to address the utility of this marker in diverse subjects and whether ancillary CT data, i.e., CACS and EFV, have different predictive value in different populations.

\section{Disclosure} interest.

Nataliya Pyslar and Rami Doukky report no conflict of

\section{References}

1. Bays HE, Gonzalez-Campoy JM, Bray GA, Kitabchi AE, Bergman DA, Schorr AB, et al. Pathogenic potential of adipose tissue and metabolic consequences of adipocyte hypertrophy and increased visceral adiposity. Expert Rev Cardiovasc Ther 2008;6:343-68.

2. Rosito GA, Massaro JM, Hoffmann U, Ruberg FL, Mahabadi AA, Vasan RS, et al. Pericardial fat, visceral abdominal fat, cardiovascular disease risk factors, and vascular calcification in a community-based sample: The Framingham Heart Study. Circulation 2008;117:605-13.

3. Nagy E, Jermendy AL, Merkely B, Maurovich-Horvat P. Clinical importance of epicardial adipose tissue. Arch Med Sci 2017;13:864-74.

4. Mazurek T, Zhang L, Zalewski A, Mannion JD, Diehl JT, Arafat $\mathrm{H}$, et al. Human epicardial adipose tissue is a source of inflammatory mediators. Circulation 2003;108:2460-6. 
5. Baker AR, Silva NF, Quinn DW, Harte AL, Pagano D, Bonser RS, et al. Human epicardial adipose tissue expresses a pathogenic profile of adipocytokines in patients with cardiovascular disease. Cardiovasc Diabetol 2006;5:1.

6. Sacks HS, Fain JN. Human epicardial adipose tissue: a review. Am Heart J 2007;153:907-17.

7. Pyslar N, Doukky R. Myocardial perfusion imaging and coronary calcium score: A marriage made in heaven. J Nucl Cardiol 2019. h ttps://doi.org/10.1007/s12350-019-01966-8.

8. Adams DB, Narayan O, Munnur RK, Cameron JD, Wong DTL, Talman $\mathrm{AH}$, et al. Ethnic differences in coronary plaque and epicardial fat volume quantified using computed tomography. Int $\mathrm{J}$ Cardiovasc Imaging 2017;33:241-9.

9. McClain J, Hsu F, Brown E, Burke G, Carr J, Harris T, et al. Pericardial adipose tissue and coronary artery calcification in the Multi-ethnic Study of Atherosclerosis (MESA). Obesity 2013;21:1056-63.

10. McClelland RL, Chung H, Detrano R, Post W, Kronmal RA. Distribution of coronary artery calcium by race, gender, and age: Results from the Multi-Ethnic Study of Atherosclerosis (MESA). Circulation 2006;113:30-7.

11. Zhou J, Liu Y, Huang L, Tan Y, Li X, Zhang H, et al. Validation and comparison of four models to calculate pretest probability of obstructive coronary artery disease in a Chinese population: A coronary computed tomographic angiography study. J Cardiovasc Comput Tomogr 2017;11:317-23.

12. Zhou J, Chen Y, Zhang Y, Wang H, Tan Y, Liu Y, et al. Epicardial fat volume improves the prediction of obstructive coronary artery disease above traditional risk factors and coronary calcium score. Circulation 2019;12:e008002.

13. Yu W, Zhang F, Liu B, Wang J, Shao X, Yang MF, et al. Incremental value of epicardial fat volume to coronary artery calcium score and traditional risk factors for predicting myocardial ischemia in patients with suspected coronary artery disease. J Nucl Cardiol 2021. https://doi.org/10.1007/s12350-021-02538-5.

14. Engbers EM, Timmer JR, Ottervanger JP, Mouden M, Knollema $S$, Jager PL. Prognostic value of coronary artery calcium scoring in addition to single-photon emission computed tomographic myocardial perfusion imaging in symptomatic patients. Circ Cardiovasc Imaging 2016;9:e03966.

Publisher's Note Springer Nature remains neutral with regard to jurisdictional claims in published maps and institutional affiliations. 